\section{Versatile light actuated matter manipulation in transparent non-dilute polymer solutions}

\author{
Manos Anyfantakis, ${ }^{* a}$ Andreas Königer, ${ }^{b}$ Stergios Pispas, ${ }^{c}$ Werner Köhler, ${ }^{b}$ Hans-Jürgen Butt, ${ }^{a}$ \\ Benoit Loppinet $^{* d}$ and George Fytas ${ }^{a d e}$
}

Received 19th November 2011, Accepted 29th December 2011

DOI: $10.1039 / \mathrm{c} 2 \mathrm{sm} 07219 \mathrm{j}$

\begin{abstract}
A local polymer concentration enhancement upon moderately weak laser irradiation was recently discovered in non-absorbing entangled polymer solutions. Here, we uncover the reverse effect: depending on the (good) solvent environment, macromolecules are either effectively attracted or repelled by visible laser light independently of the optical contrast. The versatile effect, qualitatively different from well-known mechanisms, may lead to new ways for macromolecular manipulation and micropatterning by optical fields.
\end{abstract}

Light-matter interactions have been at the focus of scientific research for centuries. Recently the class of soft materials has attracted increased attention as the utilization of light opens novel pathways to manipulate these materials on a microscopic scale. Applied optical fields can result in structural changes in complex fluids and lead to novel types of optical nonlinearities. The availability of a large variety of characteristic length scales, intermediate between atomic and macroscopic sizes, and their associated broad temporal scales ${ }^{1}$ open numerous possibilities. The detailed investigation of light-soft matter interactions has just started. ${ }^{2}$ In mixtures of materials with different polarizabilities, various sources of optical nonlinearities ${ }^{3}$ are introduced. In addition to the electronic coupling inherent to the individual components, the optical properties of the medium can change locally due to spatio-temporal variation of the local concentration $c(r, t)$. The response of $c(r, t)$, coupled possibly to temperature and molecular orientation, will therefore condition the local refractive index change $\delta n$ and, in turn, the light propagation. Various mechanisms, either well-understood like electrostriction ${ }^{4,5}$ or of less clear microscopic origin like thermo-diffusion, ${ }^{6,7}$ lead to new observations thereby adding more pieces to the puzzle of soft matter response to light irradiation., ${ }^{89}$

The effect of radiation pressure on single colloidal particles and on colloidal dispersions is well-understood and extensively used. ${ }^{5}$ The situation is somewhat less clear for macromolecules in solutions. A

${ }^{a}$ Max-Planck Institute for Polymer Research, 55128 Mainz, Germany. E-mail: anyfanta@mpip-mainz.mpg.de

${ }^{b}$ Physics Department, University of Bayreuth, 95440 Bayreuth, Germany 'Institute of Theoretical and Physical Chemistry, NHRF, Athens, Greece ${ }^{d}$ Institute of Electronic Structure and Laser, FORTH, Heraklion, Greece. E-mail: benoit@iesl.forth.gr

${ }^{e}$ Department of Materials Science and Technology, University of Crete, Heraklion, Greece series of experimental investigations on aqueous solutions of various polymers reported aggregation or phase separation induced by the application of sharply focused (diffraction limited spots) near-IR laser beams. ${ }^{10-13}$

Recently, a concentration optical nonlinearity was revealed in transparent homogeneous mixtures of alkane solvents with common polydiene polymers [cis-1,4-polyisoprene (PI) and cis-1,4-polybutadiene (PB)]. Irradiation by a weak red laser resulted in a refractive index increase $\delta n$ and the formation of polymer-rich fibers. ${ }^{14}$ This novel light-material coupling was shown to lead to a number of different patterns, such as optical spatial soliton ${ }^{15}$-like filaments, ${ }^{16}$ multi-filament arrays due to modulational instabilities ${ }^{17}$ and multifilament holographic gratings, ${ }^{18}$ depending on the irradiation conditions. ${ }^{19}$ Here, we uncover new polymer solution's responses to visible light irradiation and report on the versatility of the effect when different dispersion media are utilized.

The response of the polydiene solutions to laser light was explored with a simple experimental setup (similar to the one described in ref. 16) which allowed for their irradiation and simultaneous phase contrast (PC) imaging in the direction perpendicular to the laser beam (Fig. 1). The laser sources used were a fiber coupled diode laser (Schäfter + Kirchhoff) of wavelength $\lambda=660 \mathrm{~nm}$ and a DPSS laser (Shanghai Laser Dreams), $\lambda=671 \mathrm{~nm}$. The reported results were identical for the two wavelengths used. A microlens $(f=38 \mathrm{~mm}$, Schäfter + Kirchhoff) attached on the fiber or a microscope objective ( $f=35 \mathrm{~mm}$, Melles Griot) was used to focus the laser light in the middle of the sample cell, with focal spot diameters $d$ in the range $17-28 \mu \mathrm{m}$. The cells used were square borosilicate glass cuvettes with inner dimensions of $1 \mathrm{~mm}$ (Vitrocom) and home-made flat glass cells, mounted on a $(x, y)$ translation stage. The loading of the viscous samples was achieved by using syringes or glass pipettes. After loading, the cuvettes were carefully sealed using epoxy glue, in order to avoid solvent evaporation during the experiments. The homemade cells were sealed with a coverslip.

For the phase contrast imaging, we used the white light Köhler illumination unit of a common optical microscope (Zeiss Akioskop 2) to create a collimated beam of a diameter $d \approx 3 \mathrm{~mm}$, an $(20 \times-32 \times)$ objective lens (Zeiss) and a $640 \times 480$ CCD camera (Basler/Pixelink). A filter was placed in front of the CCD to block the scattered laser light. Images were acquired with the microscope objective lens offfocus (always above the focal plane) leading to snapshots of the intensity which can be quantitatively related to the refractive index 


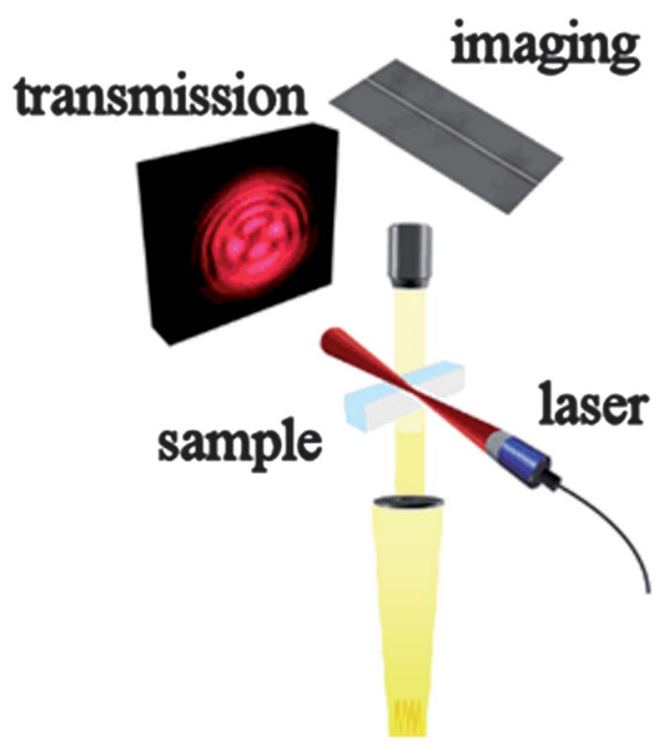

Fig. 1 A schematic of the experimental setup employed to study the response of the polydiene solutions to the application of visible laser light. The samples are irradiated and simultaneously imaged under an optical microscope in the direction perpendicular to the laser beam.

variation. $^{20}$ The imaged contrast is directly related to the difference between the local refractive index $n_{\text {str }}$ and the refractive index of the solution structure contrast $n_{\mathrm{s}}, \delta n=n_{\mathrm{str}}-n_{\mathrm{s}}$. Brighter (darker) regions in the illuminated area indicate local increase (decrease) of the index of refraction. Typical defocusing distances employed were $\Delta z=$ $20 \mu \mathrm{m}$ and $\Delta z=100-1000 \mu \mathrm{m}$ for the high contrast (i.e. Fig. 2a) and low contrast structures (i.e. Fig. 2b-d), respectively.

The samples investigated were equilibrated solutions (in the concentration range $c=0.04-0.13 \mathrm{~g} \mathrm{ml}^{-1}$ ) of anionically polymerized cis-1,4-polyisoprene $\quad\left[-\left(\mathrm{CH}_{2}-\mathrm{C}-\mathrm{CH}_{3}=\mathrm{CH}-\mathrm{CH}_{2}\right)-\right]_{N} \quad$ (PI) and

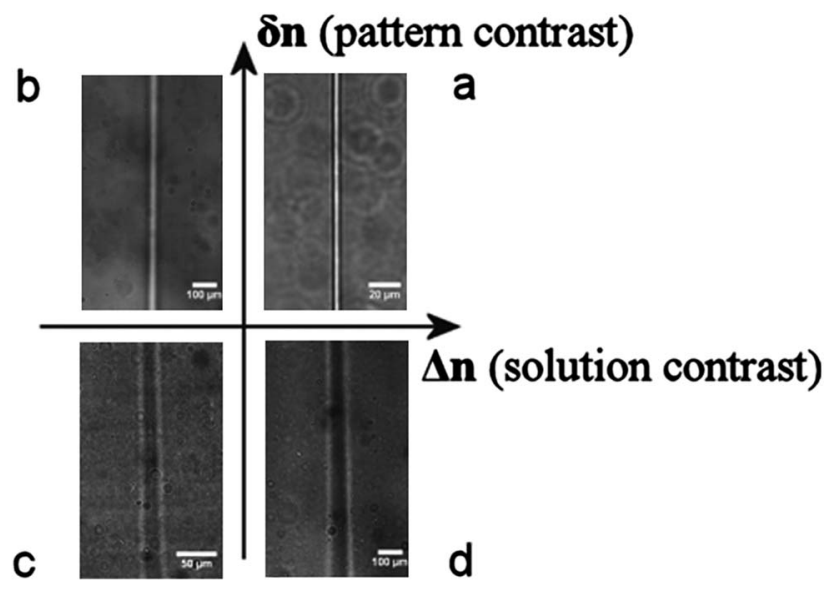

Fig. 2 The diverse light-induced micro-patterns, as imaged by phase contrast microscopy. (a) PI/decane $\left(\Delta n>0, \delta n>0, c=0.0406 \mathrm{~g} \mathrm{ml}^{-1}\right)$. (b) $\mathrm{PI} /$ bromonaphthalene $\left(\Delta n<0, \delta n>0, c=0.0413 \mathrm{~g} \mathrm{ml}^{-1}\right)$. (c) $\mathrm{PB} /$ tetrahydronaphthalene $\left(\Delta n<0, \delta n<0, c=0.0851 \mathrm{~g} \mathrm{~m}^{-1}\right)$. (d) PI/tetrahydrofuran $\left(\Delta n>0, \delta n<0, c=0.0486 \mathrm{~g} \mathrm{ml}^{-1}\right) . \Delta n=n_{\mathrm{p}}-n_{0}$ is the polymer solution optical contrast with $n_{\mathrm{p}}$ and $n_{0}$ being the refractive index of bulk polymers and the neat solvents, respectively. $\delta n=n_{\text {str }}-n_{\mathrm{s}}$ is the light induced local pattern contrast with $n_{\text {str }}$, and $n_{\mathrm{s}}$ denoting the refractive index of the illuminated (pattern) and non-illuminated (surrounding) solution. commercial cis-1,4-polybutadiene $\left[-\left(\mathrm{CH}_{2}-\mathrm{CH}=\mathrm{CH}-\mathrm{CH}_{2}\right)\right]_{N},(\mathrm{~PB}$, Polymeri Europa). The average degrees of polymerization were $N=$ 16030 and $N=7220$, and the polydispersity index PDI $=1.07$ and PDI $=2.5$ respectively. PI and PB (refractive indices $n_{\mathrm{p}}=1.519$ and $n_{\mathrm{p}}=1.52$, respectively) were dissolved in different good solvents with varying refractive index $n_{0}$ (Table 1 ), in order to vary the so-called polymer solution optical contrast, $\Delta n=n_{\mathrm{p}}-n_{0}$. All solvents were filtered through PTFE filters of pore size $0.2 \mu \mathrm{m}$ (Millipore) to ensure dust-free polymer solutions. All samples were stirred gently for several days, until homogeneous solutions were obtained.

The 'standard' effect ${ }^{14}$ is illustrated in Fig. 2a. A positive pattern contrast $\left(n_{\text {str }}>n_{\mathrm{s}}\right)$ is observed in samples with positive solution contrast, i.e. when $n_{\mathrm{p}}>n_{0}$. In this case, a polymer enriched filament is formed upon light irradiation. Polymer is attracted into the irradiated region and the formed bright filament extends over the whole cell thickness, displaying a constant diameter of about $10 \mu \mathrm{m}$. The phase contrast image in Fig. $2 \mathrm{~b}$ demonstrates the case of a negative polymer solution contrast $\left(n_{\mathrm{p}}<n_{0}\right)$. Now the solvent has a higher refractive index $\left(n_{0}=1.657\right)$ than the polymer solute and hence the observation of a positive pattern contrast $\left(n_{\mathrm{s} t r}>n_{\mathrm{s}}\right)$ implies, in this case, the formation of a polymer-depleted filament along the laser beam. Both cases, however, are in qualitative agreement with an electrostriction mechanism according to which it is the higher refractive index species being either the polymer (Fig. 2a) or the solvent (Fig. 2b) which is drawn into the inhomogeneous electric field. Based solely upon electrostrictive forces, the lower quadrants with negative pattern contrast $\left(n_{\mathrm{str}}<n_{\mathrm{s}}\right)$ should remain out of reach, as it would infer an enrichment of the lower refractive index species.

Fig. 2c and d show experimental realizations of these two unexpected cases of negative pattern contrast utilizing samples possessing both negative $\left(n_{\mathrm{p}}<n_{\mathrm{s}}=1.542\right.$, Fig. $\left.2 \mathrm{c}\right)$ and positive $\left(n_{\mathrm{p}}>n_{\mathrm{s}}=1.407\right.$, Fig. 2d) solution contrast. Indeed, Fig. $2 \mathrm{c}$ and d both show a dark stripe with low but clearly negative $\left(n_{\mathrm{str}}<n_{\mathrm{s}}\right)$ pattern contrast $\delta n$ revealing polymer enrichment and depletion, respectively. Interestingly, for the two common solvents with very close solvent refractive indices (THF and decane) used in the solutions of Fig. $2 \mathrm{a}$ and $\mathrm{d}$ with similar polymer concentration, the documented optical response is reciprocal, polymer attraction into (decane) versus repulsion from (THF) the irradiated region.

The polymer-solvent couples examined so far are presented in Table 1. Along with the signs of $\Delta n$ and $\delta n$, the solvent dipole moment $\mu$ and relative static permittivity $\varepsilon_{\mathrm{r}}$ are also shown. ${ }^{21,22}$ Neither of the mentioned physical properties define solely the response of the

Table 1 Summary of the examined solutions. The index of refraction $\left(n_{0}\right.$ at $589 \mathrm{~nm})$, the dipole moment $(\mu)$ and the relative static permittivity $\left(\varepsilon_{\mathrm{r}}\right)$ for each solvent are shown ${ }^{21,22}$

\begin{tabular}{llllll}
\hline Solvent & $n_{0}$ & $\mu(\mathrm{D})$ & $\varepsilon_{\mathrm{r}}$ & $\Delta n$ & $\delta n$ \\
\hline Hexane & 1.375 & 0 & 1.89 & + & + \\
THF & 1.407 & 1.63 & 7.52 & + & - \\
Decane & 1.411 & 0 & 1.99 & + & + \\
Cyclohexane & 1.426 & 0 & 2.02 & + & + \\
Tetradecane & 1.429 & 0 & 2.03 & + & + \\
cis-Decalin & 1.481 & 0 & 2.22 & + & + \\
Bromocyclohexane & 1.495 & 2.3 & 8.00 & + & + \\
Toluene & 1.497 & 0.375 & 2.38 & + & + \\
Chlorobenzene & 1.524 & 1.69 & 5.69 & - & + \\
1,2,3,4-Tetralin & 1.541 & 0.22 & 2.77 & - & - \\
1-Bromonaphthalene & 1.657 & 1.55 & 4.77 & - & + \\
\hline
\end{tabular}


polydiene solutions (expressed by different pattern formations) to the applied laser beam. It is important to notice that none of the polydiene solutions examined showed neutral behavior when exposed to the optical beam (which should mean no pattern formation) under the irradiation conditions mentioned so far.

Depending on the solvents, these polymer solutions respond either by an increase/decrease of both polymer concentration and refractive index independently of the solution contrast. We conclude that the sign of the polymer solution contrast $\Delta n$, which can be readily selected, does not (solely) determine the system's response to the optical field. We note that both PI and PB solutions in the same solvent displayed an identical response to light irradiation.

The minute absorption of polymer and solvent in the visible region should exclude photo-thermal and thermophoretic effects as the main driving forces. In fact, the experimental Soret coefficient for a PI/hexane solution (identical behavior to the PI/decane solution in Fig. 2a) and the PI/THF solution in Fig. 2d assumes the positive values $S_{\mathrm{T}}=0.27 \mathrm{~K}^{-1}$ and $0.046 \mathrm{~K}^{-1}$, respectively. In the presence of a temperature gradient, the polymer solute would move to the cold region, i.e. away from the laser beam in either case. Thus, the qualitative difference between the responses of the polymer solutions in neutral solvents, otherwise expected to behave similarly (a versus $\mathrm{d}$ and $\mathrm{b}$ versus $\mathrm{c}$ in Fig. 2), points towards a microscopic origin of the light-matter interaction, presumably at the atomic/molecular rather than the mesoscopic level. For completeness we want to mention that also removal of dissolved oxygen by careful degassing did not change the effect.

Moreover, the writing efficiency was found to show a strong wavelength dependence. A qualitative investigation of the laser light wavelength effect was conducted with one PI/decane solution ( $c=$ $0.04 \mathrm{~g} \mathrm{ml}^{-1}$ ) as a reference and revealed strong dependence of the formation kinetics. The response of the sample to laser light and its characteristic time was assessed either by the evolution of the transmitted beam spot or by imaging the sample under the optical microscope (Fig. 1). The light-induced concentration alteration was found to be the fastest in the red, with formation times of the order of a few seconds to a few minutes depending on the laser intensity (five wavelengths were used; $\lambda=633 \mathrm{~nm}, 647 \mathrm{~nm}, 650 \mathrm{~nm}, 660 \mathrm{~nm}$ and 671 $\mathrm{nm})$. A significant slowing down was observed for shorter wavelengths. For $\lambda=532 \mathrm{~nm}$ and $\lambda=488 \mathrm{~nm}$, the effect was present but with clearly slower response (up to $\sim 10$ times slower compared to red laser light of similar intensity). Irradiation with a near-infrared laser $(\lambda=830 \mathrm{~nm})$ did not show any change for several hours in similar conditions $(P=67 \mathrm{~mW}$, beam size similar to the one used to observe the micropatterns in Fig. 2). The light-refractive (concentration) coupling efficiency as measured by the formation kinetics is maximum in the red. This strong wavelength dependence possibly points towards a resonant mechanism.

Coupling of optical fields with matter can occur through different mechanisms. Though always related to the electronic structure, they are described at the coarse graining level of refractive index differences. In binary mixtures the sign of the light-induced concentration change is expected to be solely dictated by the sign of the polarizability or refractive index increment $(\mathrm{d} n / \mathrm{d} c)$ through the electrostriction mechanism. ${ }^{23}$ Simple descriptions in terms of refractive indices, however, oversimplify the local solute-solvent interactions. This macroscopic light-matter coupling cannot capture, even qualitatively, the observed behavior in Fig. $2 \mathrm{a}-\mathrm{d}$, where similar polymer solution optical contrasts lead to opposite behaviors.
In summary, we have shown an effect of light-induced attraction or repulsion in polydiene-based non-dilute solutions, with clear wavelength dependence on the kinetics. In particular we have presented cases of micropatterns with refractive index lower than the average refractive index of the material, which is against an electrostrictive mechanism. Such a behavior is unexpected for transparent binary solutions. Polydiene solutions in good solvents therefore provide media with a unique type of light-refractive index coupling. This new type of photonic materials may offer a rich platform for the investigation of light-matter interactions with an un-equaled versatility. Depending on the precise conditions (material and irradiation) light application can give rise to a large number of light propagation and material patterning effects. This effect could be utilized to manipulate and pattern transparent polymer solutions at moderately low laser intensities.

Shedding light on the specificity of these materials might unlock the quest for other complex fluids which could present similar phenomena. Elucidation of the underlying mechanism might facilitate the control of macromolecular manipulation, writing of three dimensional structures and the investigation of soft matter nonlinear optics taking advantage of the unique versatility of polymers.

The Greek Secretariat for Research and Technology (program PENED-03ED805), the Max Planck Society (program IMPRS for polymer materials), and the DFG (Bu 1556/27) are acknowledged for the financial support. Dr Christos Mantzaridis' help with polymer synthesis is also acknowledged.

\section{Notes and references}

1 R. A. L. Jones, Soft Condensed Matter, Oxford University Press, New York, 2002.

2 C. Conti, G. Ruocco and S. Trillo, Phys. Rev. Lett., 2005, 95, 183902.

3 G. I. A. Stegeman, D. N. Christodoulides and M. Segev, IEEE J. Sel. Top. Quantum Electron., 2000, 6, 1419-1426.

4 A. Ehrlicher, T. Betz, B. Stuhrmann, D. Koch, V. Milner, M. G. Raizen and J. Käs, Proc. Natl. Acad. Sci. U. S. A., 2002, 99, 16024.

5 A. Ashkin, Science, 1980, 210, 1081-1088.

6 S. Duhr and D. Braun, Proc. Natl. Acad. Sci. U. S. A., 2006, 103, 19678-19682.

7 W. Köhler, A. Krekhov and W. Zimmermann, Adv. Polym. Sci., 2010, 227, 145-198.

8 C. Conti and E. Del Re, Phys. Rev. Lett., 2010, 105, 118301.

9 P. J. Reece, E. M. Wright and K. Dholakia, Phys. Rev. Lett., 2007, 98, 203902.

10 P. Borowicz, J.-I. Hotta, K. Sasaki and H. Masuhara, J. Phys. Chem. $B, 1997, \mathbf{1 0 1}, 5900-5904$.

11 J. Hofkens, J.-I. Hotta, K. Sasaki, H. Masuhara, H. Faes and F. C. De Schryver, Mol. Cryst. Liq. Cryst. Sci. Technol., Sect. A, 1996, 283, 165-172.

12 T. A. Smith, J.-I. Hotta, K. Sasaki, H. Masuhara and Y. Itoh, J. Phys. Chem. B, 1999, 103, 1660-1663.

13 Y. Tsuboi, M. Nishino, Y. Matsuo, K. Ijiro and N. Kitamura, Bull. Chem. Soc. Jpn., 2007, 80, 1926-1931.

14 R. Sigel, G. Fytas, N. Vainos, S. Pispas and N. Hadjichristidis, Science, 2002, 297, 67-69.

15 G. I. Stegeman and M. Segev, Science, 1999, 286, 1518-1523.

16 M. Anyfantakis, B. Loppinet, G. Fytas and S. Pispas, Opt. Lett., 2008, 33, 2839-2841.

17 D. Kip, M. Soljacic, M. Segev, E. Eugenieva and D. N. Christodoulides, Science, 2000, 290, 495-498.

18 B. Loppinet, E. Somma, N. Vainos and G. Fytas, J. Am. Chem. Soc,, 2005, 127, 9678-9679.

19 M. Anyfantakis, G. Fytas, C. Mantzaridis, S. Pispas, H.-J. Butt and B. Loppinet, J. Opt., 2010, 12, 124013.

20 A. Barty, K. A. Nugent, D. Paganin and A. Roberts, Opt. Lett., 1998, 23, 817.

21 CRC Handbook of Chemistry and Physics, ed. D. R. Lide, CRC Press, 2009.

22 Handbook of Organic Solvents, ed. D. R. Lide, CRC Press, 1995.

23 Y. Tsori, F. Tournilhac and L. Leibler, Nature, 2004, 430, 544. 